\title{
Los parásitos de la ciencia. Una caracterización psicocognitiva del engaño pseudocientífico
}

\author{
(The Parasites of Science. \\ A Psycho-cognitive Characterization of a Pseudo-scientific Hoax)
}

\author{
Angelo FASCE
}

Recibido: 27/04/2017

Versión final: 16/06/2017

BIBLID 0495-4548(2017)32:3p.347-365

DOI: $10.1387 /$ theoria. 17775

RESUMEN: El marco teórico desde el cual se llevan a cabo investigaciones acerca de la pseudociencia es deficiente, dado que suele incluir otros tipos de creencias carentes de garantía epistémica. En este artículo, se repasarán los mecanismos de explotación de la autoridad científica por parte de la pseudociencia, desarrollando así un marco psicocognitivo más refinado para caracterizar el fenómeno. Se analizará la psicología del engaño pseudocientífico, las raíces cognitivas que posibilitan la epidemiología de este tipo de ideas y sus mecanismos de autolegitimación, como la superioridad dialéctica, el falso apoyo externo o la falsa superioridad ética y/o epistemológica.

Palabras clave: Pseudociencia, demarcación, autoridad científica, garantía epistémica.

ABSTRACT: The theoretical framework of research on pseudoscience is deficient, since it usually includes other types of beliefs lacking epistemic warranty. In this paper, I review the mechanisms of exploitation of scientific authority by pseudoscience, developing a more refined psycho-cognitive framework to characterize the phenomenon. I will analyze the psychology of pseudoscientific deception, the cognitive roots that enable the epidemiology of these ideas and their mechanisms of self-legitimation, such as dialectical superiority, false external support or false ethical and/or epistemological superiority.

Keywords: Pseudoscience, demarcation, scientific authority, epistemic warranty.

La pseudociencia constituye una forma de fraude intelectual consistente en ideas ajenas a la ciencia que, en un intento de aumentar su prestigio y aceptación social, se impostan como científicas. Por ello, además de constituir creencias carentes de garantía epistémica, consiste en un engaño con profundas implicaciones sociales y académicas que se ha infiltrado en casi todos los ámbitos de estudio y práctica científica. Esta característica definitoria de la pseudociencia, el impostarse como ciencia, queda plasmada tanto en la propia etimología del término como en el criterio de demarcación propuesto por Sven Ove Hansson (2009; 2013), una propuesta demarcativa que, aunque resulta ambigua en su formulación, ofrece un marco de referencia que se corresponde con la definición intuitiva y más habitual de la 
pseudociencia. El criterio de Hansson establece que un enunciado es pseudocientífico si y solo si:

1. Se refiere a un problema dentro del dominio de la ciencia en un sentido amplio (criterio de dominio científico).

2. Adolece de una grave falta de fiabilidad, tal que no resulta en absoluto ser de confianza (criterio de la falta de fiabilidad).

3. Es parte de una doctrina para la que sus defensores tratan de crear la impresión de que representa el conocimiento más confiable de su temática (criterio de la doctrina desviada) (Hansson 2013, 71-72).

En consecuencia, una propuesta, ya sea teórica o práctica, constituirá una pseudociencia si y sólo si cumple los dos primeros puntos y, además, se imposta como ciencia. Los ejemplos que cumplen estas condiciones son numerosos, y se instalan en campos como la biología -lysenkoísmo, Diseño Inteligente-, la medicina — homeopatía, quiropraxia, terapia neural一, la psicología — psicoanálisis, hipnosis regresiva, programación neurolingüística一, los productos milagro - cremas con ADN, la dieta del grupo sanguíneo-, o la historia - negacionismo del Holocausto, astronautas de la antigüedad. En este sentido, el tercer punto resulta definitorio, siendo aquel que traza la línea que separa otras creencias irracionales, como pueden ser las teorías conspirativas o el pensamiento paranormal, de la pseudociencia. Sin embargo, pese a su gran prevalencia social y a que conocemos bien las dinámicas de otros tipos de creencias carentes de garantía epistémica (Lindeman y Aarnio 2006; Brotherton, French y Pickering 2013), lo cierto es que las características psicológicas y sociológicas del engaño pseudocientífico han sido escasamente estudiadas desde un punto de vista académico. Uno de los principales problemas relativos al estudio de la pseudociencia reside en el marco teórico poco desarrollado que presenta, de modo que mientras la caracterización del pensamiento paranormal y conspirativo es sofisticada, teniendo incluso sus propias escalas de medición psicológica debidamente validadas (Tobacyk 2004; Brotherton, French y Pickering 2013), el estudio de la pseudociencia tiene grandes problemas de cribado y carece de una escala propia que resulte adecuada.

En los escasos artículos académicos que investigan la prevalencia de la pseudociencia y sus predictores psicológicos podemos encontrar marcos teóricos que carecen de la aplicación de un criterio de demarcación funcional. Por ejemplo, en (Majima 2015), encontramos como parte de lo que el autor denomina «pseudociencia paranormal» las mancias o la creencia en Dios. La religiosidad, sin embargo, suele listarse dentro de la psicología entre las creencias paranormales (Tobacyk 2004). Por su parte, entre lo que denomina «pseudociencia no-paranormal» encontramos mitos como los supuestos eventos extraños en el comportamiento animal y el retroceso del nivel del agua del océano antes de un terremoto o el mito que asegura que escuchar a Mozart aumenta el nivel de inteligencia de los niños - una idea que suele ser listada en los estudios sobre psicomitos (Hughes, Lyddy y Lambe 2013; Gaze 2014). El nivel de pseudocientificidad, de impostación como ciencia, de estas cuestiones no permite que todas las creencias y prácticas listadas por Majima puedan ser consideradas como un conjunto más o menos homogéneo que sirva como campo de estudio elucidado.

Lundstrôm y Jakobsson (2009) incluyen como pseudociencia la telepatía, la telequinesis, la influencia de las fases lunares en nuestra conducta o el uso de péndulos para decidir el sexo de los bebés. Todas estas ideas suponen el $40 \%$ del total de la escala que emplean en su estudio - a lo que se podría sumar un ítem más que hace referencia a la astrología, cuyo estatus 
como pseudociencia en la actualidad en controvertido, dado que también suele listarse dentro de las creencias paranormales (Tobacyk 2004). En Tseng et al. (2013) encontramos un problema parecido. Su escala, dejando de lado el feng-shui y la astrología como casos límite, contiene un $60 \%$ de contenido perteneciente al pensamiento paranormal y no propiamente pseudocientífico — por ejemplo, números de la suerte, precognición o telequinesis.

Hay casos de marcos teóricos aún más carentes de solidez, como (Franz y Green 2013), en el que el $100 \%$ de los contenidos de las escalas empleadas son propios de pensamiento paranormal o espiritual. O como (Johnson y Pigliucci 2004), que incluyen en su escala de creencias pseudocientíficas, dejando de lado nuevamente a la astrología, un $80 \%$ de ítems que hacen referencia al pensamiento paranormal o conspirativo —alienígenas en el área 51, la supuesta capacidad de los animales para detectar fantasmas, el vudú o creer que romper un espejo da mala suerte. La situación es grave, dado que se están publicando estudios que afirman medir la pseudociencia cuando se está midiendo cuestiones de otro tipo en base a considerar la definición de «pseudociencia» desde el punto de vista del mero parecido de familia (Pigliucci 2012). El problema se vuelve aún más grave si observamos casos en los que se pone bajo la misma agrupación conceptual a la homeopatía y a la quiropraxia con la ciencia cognitiva, la sociobiología o la memética (Bunge 1985).

Resulta una tarea de gran importancia entonces el desarrollo de un marco conceptual suficientemente potente, que sea capaz de elucidar el tercer punto del criterio de Hansson, a fin de aumentar la capacidad de los investigadores, de los pensadores escépticos y de los consumidores para cribar entre la pseudociencia y otras creencias carentes de garantía epistémica. Con el objetivo de examinar los engranajes de sus mecanismos de explotación de la ciencia, separaré la siguiente exposición en tres esferas de análisis: la psicología del pseudocientífico, las bases cognitivas de la pseudociencia y sus mecanismos efectivos de actuación. Por ello, dedicaré un apartado inicial a la caracterización de la psicología del fraude pseudocientífico. Tras esta cuestión, que subyace al fenómeno sociológico y psicológico que supone la pseudociencia, abordaré sus causas últimas: las raíces cognitivas que posibilitan la epidemiología de la pseudociencia. Finalmente, abordaré las causas próximas de la pseudociencia, sus mecanismos efectivos de impostación como ciencia, que dividiré en tres apartados: «superioridad dialéctica», «falso apoyo externo, falsas credenciales» y «falsa superioridad moral y epistemológica».

\section{La psicología del engaño pseudocientifico}

Como bien se indica en el esquema general de Hansson, la clave de la pseudociencia reside en el prefijo «pseudo», que indica que se trata de «falsa ciencia». En este sentido, es un fraude por propia definición. Si la pseudociencia constituye una forma de mentira, y no únicamente de engaño, en todas sus instancias es algo que resulta complejo de determinar, dado que supondría valorar estados psicológicos relativos a creencias y a grados de honestidad, lo cual puede resultar imposible incluso a nivel tecnológico. Por ello, llevar el reconocimiento de la pseudociencia al reconocimiento efectivo de la consciencia de la mentira supone una táctica muy poco funcional; es posible que estas modificaciones conscientes en la doctrina convivan con cambios que tengan lugar debido a la selección de mutaciones más o menos azarosas de ideas anteriores. El estudio de la pseudociencia puede, y debe, combinar ambos casos. 
La capacidad del ser humano para la de detección inmediata de engaños es tan baja que se acerca al azar (Bond y DePaulo 2006), con una pequeña fracción de aciertos por encima de lo aleatorio que se debe más a malos engañadores que a buenos detectores (Levine 2010) -a lo que hay que sumar fenómenos como, por ejemplo, las falsas memorias (Roediger y McDermott 1995; Braun, Ellis y Loftus 2002). El principal problema es que las personas tienden a fijarse en el lenguaje corporal, en el nerviosismo (The Global Deception Research Team 2006) o en la actitud general de la persona, de un modo bastante prejuicioso y estereotipado (Levine et al. 2011), cuando esta clase de indicadores son poco fiables. Ello lleva a que ciertas personas sean consideradas sospechosas habituales de mentir siendo inocentes y a otras que, mintiendo pero controlando estos indicadores intuitivos, son capaces de aparentar la más incorruptible honestidad. Pero, pese a estas dificultades para captar engaños en un primer momento, existen determinados mecanismos que permiten a las víctimas de engaños desenmascararlos a posteriori - por ejemplo, el testimonio de terceras personas o la evidencia empírica- (Park et al. 2010).

Es comprensible que el ser humano no haya desarrollado mecanismos fiables para detectar engaños guiándose por indicadores visibles en el momento mismo de mentir, dado que la interacción por defecto en las relaciones humanas es la sinceridad (Levine, Kim y Hamel 2010) y, por ello, la gente suele pensar por defecto que su interlocutor está siendo sincero (Levine 2014). De hecho, se calcula que solo el 5\% de la población de los EEUU es culpable del 50\% de las mentiras totales del país (Serota, Levine y Boster 2010), y que la población del Reino Unido tiene únicamente una tasa de 0,41 mentiras al día (Serota y Levine 2014); unos niveles de mentirosos y de mentiras que se mantienen de forma intercultural (Mann et al. 2016). Entonces, ¿̨por qué no miente más la gente? Esta baja tasa se debe a que, si bien la motivación para la mentira puede ser grande, la honestidad también goza de potentes motivadores sociales. Sin ir más lejos, la honradez es uno de los valores más apreciados socialmente (CIS 2001), de modo que se tiende a sentir una gran animadversión hacia el que miente a los demás. Esta realidad contrasta con los beneficios sociales y personales del autoengaño, dado que las personas con niveles poco realistas de confianza en sí mismas tienen mayor atractivo sexual (Murphy et al. 2015) - esos niveles correlacionan con niveles altos de testosterona (Ronay 2016) - y éxito social (Anderson 2012). El autoengaño suele ser una herramienta muy útil para convencer a los demás (von Hippel y Trivers 2011), dado que hace sentir bien a esa persona, genera motivación en ella y proyecta una imagen de liderazgo y seguridad que la convierte en más eficaz engañando a los demás (Lamba y Nityananda 2014; Smith, Trivers y von Hippel. 2017).

La principal motivación que pueda tener la gente para no mentir o engañar y, con ello, para no parasitar el prestigio de la ciencia, es el mantenimiento de la autoimagen (Mazar, Amir y Ariely 2008). Si bien existen pocos controles sobre los practicantes de las pseudociencias, y un acceso sencillo a una retribución monetaria, es la ruptura con la autoimagen de aquel que lleva a cabo estas prácticas el principal motivador para la honradez. Al fin y al cabo, una oferta de libre encuentro sexual en un entorno ajeno a la prostitución puede ser más o menos bien recibida, pero la misma oferta incluyendo un intercambio de dinero bajaría considerablemente las opciones de que se llegue a materializar. Este tipo de casos suponen claros contraejemplos a los modelos económicos más reduccionistas, en los que los individuos únicamente buscan la maximización del beneficio económico, cuando lo cierto es que la mera suma de motivadores, o la mera presencia de dinero, no significa, necesariamente, un mayor nivel de motivación para el comportamiento prosocial (Ariely, Brach y Meier 2009). 
Las personas, de hecho, suelen mentir únicamente hasta un nivel en el que puedan ser capaces de salvar su propia autoimagen de integridad, racionalizando para ello sus conductas inmorales (Tsang 2002). Hay dos mecanismos principales en este proceso (Mazar, Amir y Ariely 2008): 1) la desatención a los propios estándares morales y 2) la maleabilidad en la categorización de la mentira. Los sistemas pseudocientíficos han de facilitar el proceso de racionalización de sus adeptos, facilitando la aparición de disonancias cognitivas (Festinger 1957) y de auto-afirmaciones (Steele 1998). De este modo, han de ser capaces de generar contextos en los que estos dos mecanismos puedan ser llevados a cabo con facilidad a fin de que sostener dichas ideas no atente directamente contra la autoimagen de los pseudocientíficos, especialmente en los casos en que sus creencias afectan de forma negativa a la integridad física de otras personas. En este sentido, neutralizar la identidad moral es algo clave para la supervivencia de una pseudociencia, lo cual viene mediado, entre otras cosas, por la supresión de la atención en los códigos deontológicos de la educación o de las profesiones sanitarias, o por una actitud victimista y conspirativa en la que la industria y el sistema serían la fuente de un sufrimiento mucho mayor.

Todas estas ideas arraigan con tal fuerza en el sistema conceptual de los pseudocientíficos que está documentado el backfire effect en su acercamiento a la evidencia científica (Lord, Ross y Lepper 1979); es decir, el hecho de que, en ocasiones, exponerlos a ella puede ser contraproducente en aras de que abandonen sus concepciones erróneas de la realidad (Nyhan y Reifler 2015). El pseudocientífico se ve a sí mismo como un luchador social, y tiene a mano una gran cantidad de herramientas, bajo la forma de sistemas de ideas autovalidantes (Boudry y Braeckman 2012) o de hipótesis ad-hoc, que permiten racionalizar y aumentar sus niveles de disonancia cognitiva, convirtiendo sistemáticamente las críticas y refutaciones en confirmaciones respecto a sus ideas y a su autoimagen. El problema de esta autoconcepción de luchador social es que motiva para engañar más a los demás, al creer que está haciendo el bien con ello (Gino, Ayal y Ariely 2013).

Una de las consecuencias que pueden sacarse de este análisis de las bases psicológicas del engaño pseudocientífico es que deberían ser buenas técnicas de contención del fenómeno, tanto el reconocimiento constante de los códigos éticos y el mantenimiento de la identidad moral de la población, como el hecho de que el engaño suele proceder generalmente de las personas creativas, al ser mejores generando relatos autojustificantes. Ambas cuestiones, de hecho, están avaladas por la evidencia disponible. Las personas creativas mienten con más frecuencia (Gino y Ariely 2011). No tanto las más inteligentes —definiendo la inteligencia como la habilidad general para resolver problemas-, sino los denominados «pensadores originales»; aquellos pensadores con mayor capacidad para inventar relatos, con más flexibilidad social e incluso con más materia blanca - mayor interconexión entre áreas cerebrales. Por otro lado, cuando la gente engaña a otra en contra de sus principios morales se genera en ellos lo que los psicólogos denominan «disonancia ética». Inhibir la capacidad de los individuos para justificar este tipo de disonancia disminuye su tendencia a comportarse de un modo inmoral (Barkan, Ayal y Ariely 2015).

Sabemos que las personas que son expuestas a códigos éticos, como, por ejemplo, los mandamientos cristianos, reducen su tasa de mentiras (Mazar, Amir y Ariely 2008) - incluso aunque no consideren que ese sea su propio código ético. Mantener viva la llama de la ética resulta muy importante, porque cada político, médico, compañero o familiar corrupto que saca beneficio del engaño genera la idea de que ser honesto es algo que no vale la pena, lo cual resulta peligroso porque se miente más cuando se detecta que es una actitud acep- 
tada en el entorno (Gino, Ayal y Ariely 2009; Mann et al. 2016). Jugar con la propia reputación es algo que siempre debería estar altamente penalizado en nuestras interacciones sociales, de modo que adherirse a la pseudociencia no debería dejar nunca de ser algo que conlleve grandes costes sociales.

Con todo lo dicho, el ambiente adecuado, que debemos prevenir, para que el engaño pseudocientífico sea rentable y psicológicamente exitoso es el siguiente:

1. Alta recompensa tras el engaño.

2. Mantenimiento de la autoimagen tras adherirse a las ideas pseudocientíficas.

3. Posibilidad de autojustificación creativa ante el daño causado.

4. Poca respuesta punitiva de los demás ante 1,2 y 3 .

\section{Causas últimas: las raices cognitivas de la pseudociencia}

Las dinámicas de las creencias pseudocientíficas se diferencian sustancialmente de otro tipo de creencias carentes de garantía epistémica, como aquellas paranormales o conspiranoicas, aunque la investigación específica aún necesita avanzar. Hay, por ejemplo, una gran cantidad de estudios sobre adeptos a las terapias alternativas y complementarias (CAM), pero dichos estudios no correlacionan de forma fiable las creencias pseudocientíficas en relación a ciertas variables psicológicas que pudieran servir como predictores, porque dentro de las CAM se contabilizan pseudoterapias pseudocientíficas y no pseudocientíficas (Quandt et al. 2009). Un estudio especialmente interesante fue el llevado a cabo por Emilio Lobato y sus colaboradores en la Universidad Central de Florida (Lobato et al. 2014). Este estudio tiene la peculiaridad de comparar entre sí los tres grupos clásicos de creencias sin garantía epistémica - pseudocientíficas, conspirativas y paranormales. Lo que Lobato y sus colaboradores encontraron fue que las creencias paranormales y las conspirativas tienen un perfil psicológico relativamente parecido, correlacionando entre ellas y teniendo como mejor predictor lo que se denomina en psicología «confusiones ontológicas». Estas confusiones consisten en la tendencia a confundir entre sí las propiedades de los niveles físico, biológico y psicológico, de modo que, por ejemplo, se atribuyen sentimientos a objetos inanimados - aunque las creencias paranormales tenían, además, una correlación fuerte con el neuroticismo y las conspirativas una correlación débil con el deseo de control.

Las creencias pseudocientíficas, por su lado, no correlacionaron con estas confusiones ontológicas. La pseudociencia, en cambio, presentó correlación con las creencias conspirativas, una correlación algo menor con las creencias paranormales y correlación con la pertenencia religiosa. La correlación con el pensamiento conspirativo parece acrecentarse en los casos de pseudociencia negacionista (Lewandowsky, Gignac y Oberauer 2013; Lewandowsky, Oberauer y Gignac 2013), siendo moderada con la doctrinal (Hansson 2017). Por su parte, la correlación con el pensamiento paranormal apoya la tesis del pensamiento monológico respecto a los tres tipos de creencias sin garantía epistémica. Sin embargo, no se trata de evidencia concluyente, dado que también disponemos de evidencia en favor de un efecto sustitutivo entre el pensamiento paranormal y el pseudocientífico (Losh y Nzekwe 2011). Por último, es posible interpretar que la correlación con la religiosidad responde a un mal diseño de la escala empleada para medir las creencias pseudocientíficas; una escala $a h$-hoc que incluía entre sus ítems de forma recurrente al Diseño Inteligente, una pseudo- 
ciencia estrechamente relacionada con la derecha religiosa de los Estados Unidos. Sin embargo, los resultados del análisis de datos del estudio de Lobato no dejan lugar a dudas: la pseudociencia ocupa un lugar muy particular dentro del grupo de creencias sin garantía epistémica. La razón de esta situación es sencilla de explicar si tenemos en cuenta que, a diferencia de los otros dos tipos, la pseudociencia parasita a la ciencia, impostándose como tal. Por ello, sus adeptos son, en realidad, adeptos a la ciencia; sienten un respeto paradójico por ella y prefieren sus estándares de fiabilidad, pero mantienen una visión conspirativa respecto a las instituciones científicas.

Se han llevado a cabo varias teorizaciones acerca de la epidemiología de las ideas pseudocientíficas (Boudry et al. 2014), analizando los elementos que le darían el enorme poder de expansión social que presenta, así como sus características sociológicas - por ejemplo, explicando su poca presencia en zonas donde la ciencia no goza de prestigio social. El entorno cultural que explota la pseudociencia puede ser definido del siguiente modo: «Los mecanismos de vigilancia epistémica, una atmósfera en la que la ciencia es considerada como una autoridad epistémica, y un público que carece de una correcta comprensión de dicha autoridad, todo ello junto supone las condiciones suficientes para que emerja la pseudociencia» (Blancke, Boudry y Pigliucci 2016). De este modo, hay 1) una población que aplica mecanismos de vigilancia a fin de no ser engañada, que en este caso se materializa en confianza en la ciencia; 2) una incorrecta valoración de la autoridad científica; y 3) una parte de la población que explota tanto la voluntad de adherirse a la ciencia como la ignorancia acerca de su autoridad.

En psicología está fuertemente contrastado el modelo dual de la cognición humana, en el que existen dos sistemas de procesamiento de la información que funcionan en paralelo y que suponen subsistemas diferenciados a nivel cerebral (Tsujii y Watanabe 2009): el sistema intuitivo y el analítico (Epstein et al. 1996). El sistema intuitivo es rápido e inherentemente sesgado, permitiéndonos tomar decisiones inmediatas y poco razonadas, mientras que el analítico es lento, costoso y basado en el pensamiento crítico. Por ejemplo, las creencias paranormales correlacionan negativamente con el pensamiento analítico (Lindeman y Aarnio 2006), mientras que el ateísmo lo hace positivamente (Gervais y Norenzayan 2012). La pseudociencia, en este sentido, constituiría un punto intermedio entre ambos estilos cognitivos, al consistir en un intento de hacer pasar como analíticas creencias intuitivas. De hecho, se sabe que la relación entre la pseudociencia y el sistema analítico de pensamiento es problemática (Majima 2015). Tampoco existe una correlación directa entre mayores conocimientos científicos y menor creencia en la pseudociencia (Walker et al. 2002; Johnson y Pigliucci 2004; Majima 2015). Parece evidente, entonces, que la detección de la pseudociencia requiere de una serie de nociones epistemológicas muy específicas y no únicamente de presentar pensamiento analítico o de poseer conocimientos acerca de teorías científicas, algo que ha llevado a plantear la necesidad de enseñar de un modo explícito, también a los científicos, a detectar pseudociencias (Lilienfeld, Lohr y Morier 2004; Pigliucci 2007).

Blancke, Boudry y Pigliucci (2016) hacen referencia a dos mecanismos cognitivos como los principales culpables de que el uso de la ciencia como un argumento constituya un mecanismo altamente eficaz de expansión para las ideas sin garantía epistémica: la vigilancia epistémica y la valoración de la autoridad. La vigilancia epistémica es definida como un mecanismo cognitivo consistente en la capacidad para evadir la desinformación o para evitar aprender comportamientos no adaptativos (Sperber et al. 2010). Se trata de varios mecanismos que alertan a las personas acerca de la presencia de engaños en el discurso de sus inter- 
locutores, capacitándolas para detectar la verdadera experticia y la información más fiable en un momento dado - una capacidad que se desarrolla a lo largo de la infancia, en paralelo a las habilidades relacionadas con la capacidad de colaboración y con la empatía cognitiva (Mascaro y Sperber 2009).

Las investigaciones de Sperber et al. (2010) han sido especialmente esclarecedoras en este sentido, considerando que esta vigilancia puede ser focalizada tanto hacia el mensaje como hacia el interlocutor. En el caso de la ciencia, la evaluación del mensaje es compleja, porque se trata de un discurso que resulta tremendamente antiintuitivo y complejo, que demanda una extensa y costosa formación para adquirir la capacidad para comprenderlo. Por esta razón, la vigilancia epistémica respecto a las afirmaciones científicas no suele ser llevada a cabo tanto en relación al mensaje como en relación a la fiabilidad de la fuente de información, a su aparente honestidad y competencia. La mayor parte de la población, debido al tipo de educación que recibe y a las limitaciones intrínsecas que la especialización científica impone, únicamente es capaz de valorar la ciencia en relación a las características del interlocutor y a su apariencia estereotipada de cientificidad. Esto ha llevado a una comprensión muy deficiente de la naturaleza de la ciencia (Sumranwanich y Yuenyong 2014), incluso entre profesores de ciencia (Alswelmyeen y Al olimmat 2013).

Este déficit en la comprensión de la naturaleza de la ciencia lleva, a su vez, a una profunda incomprensión de la autoridad científica, de modo que, sin comprender qué es la ciencia, su autoridad acaba siendo entendida como un conjunto de características psicológicas o sociológicas y no como la capacidad efectiva de comprensión y comunicación de evidencia científica. La autoridad científica tiene una naturaleza diferente a otros tipos de autoridad que valoramos en nuestro día a día; autoridades informales que mantienen un alto contenido retórico y autoritario, y que se relacionan estrechamente con el carisma, la legitimidad social o la capacidad de convicción. Los mecanismos de legitimación de la autoridad extra-científica han sido profusamente estudiados por la psicología (Tyler 2006), y, pese a que son mecanismos complejos, muchas veces pueden venir mediados por cuestiones tan prejuiciosas y sencillas como el atractivo físico (Praxmarer 2011), la sonrisa (Schmidt, Levenstein y Ambadar 2012) o la fama (Kaikati 1987), una serie de sesgos que pueden verse aún más agudizados en el caso de la atribución de autoridad vía internet (Metzger y Flana$\operatorname{gin} 2013)$.

Alvin Goldman localiza tres fuentes de valoración informal de la autoridad (Goldman 2001). En primer lugar, el análisis de los argumentos desde un punto de vista de la «superioridad dialéctica». Esta superioridad es muy diferente dentro y fuera de los ambientes que practican el pensamiento crítico, dado que en los contextos menos ilustrados suele hacer referencia a la capacidad retórica para convencer, independientemente de la validez de los argumentos. En segundo lugar, el apoyo que tiene tal o cual experto por parte de otros expertos. El apoyo externo es algo que tiene un fuerte impacto psicológico en el oyente, e incluso la ciencia exige entre sus estándares argumentativos la apelación a citas que hagan referencia al trabajo de otras personas y que apoyen el propio - algo que puede ser un arma de doble filo, dado que puede suponer también un contratiempo respecto al acceso que ideas novedosas o heterodoxas puedan tener en publicaciones científicas de prestigio. En tercer lugar, el valor de las credenciales del supuesto experto. Valorar sus titulaciones, el prestigio de las universidades que las han expedido, su renombre, su jerarquía dentro de la institución en la que trabaja, etc. Y, por último, el análisis de los posibles sesgos o conflictos de intereses que puedan afectar de forma grave a la credibilidad o a la integridad ética del interlocutor. 
La pseudociencia explota esta valoración intuitiva del experto, intentando que la evaluación de la experticia científica sea llevada a cabo según estándares informales y no según los estándares propios de la ciencia. Una vez consigue este efecto, deslizando a sus adeptos hacia una valoración de la autoridad científica equivalente a la que llevamos a cabo con la autoridad empresarial o política, resulta un mero ejercicio de mercadotecnia el impostarse atendiendo a los cuatro factores que Goldman apunta. Una tarea para la cual, además, los pseudocientíficos están más preparados que los propios científicos, que habitualmente no necesitan preocuparse por estas cuestiones. En ciencia es irrelevante la posición o las titulaciones o el carisma en relación a la justificación que pueden tener otros científicos para creer en lo que se defiende - o, al menos, así debería ser desde un punto de vista normativo-, teniendo como obligación epistémica atender únicamente a la evidencia científica disponible. Sin embargo, el impacto psicológico, a nivel social, de las ideas de alguien que emane autoridad en el sentido de prestigio, de apoyo social, de supuesta falta de intereses ocultos e incluso de una valencia positiva en el nivel emocional y moral respecto a sus ideas es mucho mayor que el impacto de aquel que pretende legitimarse con base en un discurso seco, basado en estudios que pueden resultar incomprensibles y que, además, ofrecen al lego la a veces desagradable sensación de ignorancia y de falta de cierre epistémico.

Los pseudocientíficos explotan la autoridad científica a fuerza de desvirtuarla, ofreciendo una explicación paralela a los fenómenos que resulta altamente intuitiva, de acceso mucho más sencillo por parte del oyente (Blancke et al. 2015). El psicoanálisis es más intuitivo que la terapia cognitiva-conductual, la homeopatía es más intuitiva que la medicina, el Diseño Inteligente lo es más que la teoría de la evolución. Mientras que la ciencia va a contrapelo de los sesgos cognitivos que nos afectan, la pseudociencia los tiene como viento de cola. Las personas, además, muestran conformismo ante la autoridad (Michener y Burt 1975), incluso en casos extremos que las llevan a cometer actos que rompen de forma radical con sus preceptos morales (Milgram 1963). A esto se suma la dependencia que generan entre sus adeptos los sistemas pseudocientíficos, algo que genera que la autoridad sea valorada de un modo mucho mayor (van der Toorn, Tyler y Jost 2011). Por ejemplo, generan dependencia emocional respecto a la gran cantidad de sesiones que les programan a sus clientes o a los supuestos eventos catastróficos que ocurrirán en sus vidas si abandonan dichas sesiones. Además, claro, de la dependencia intelectual, moral y de todos los supuestos beneficios que sus adeptos reciben como miembros de un grupo social que se organiza en torno a una serie de ideas o a un gurú - siendo el caso del gurú bastante habitual, dada nuestra tendencia a confiar en una única figura de autoridad cognitiva en lugar de en varias (Schwartz, Luce y Ariely 2011).

\section{Causas próximas: la ciencia como argumento}

La pseudociencia emplea a la ciencia como argumento. La ciencia argumenta sobre la base de la evidencia — «esta teoría goza de amplio respaldo evidencial»-, mientras la pseudociencia se limita a argumentar sobre la base de la ciencia misma, entendida como una muestra de estatus — «esta teoría es científica». Para generar este tipo de apariencia de autoridad, la pseudociencia utiliza tres estrategias principales, que son la superioridad dialéctica, el falso apoyo externo, y la falsa superioridad moral y/o epistemológica. Las tres estrategias se relacionan estrechamente con las fuentes de autoridad de Goldman, de modo que son capaces de desactivar los mecanismos de vigilancia epistémica de la población. 


\section{LA SUPERIORIDAD DIALÉCTICA}

La retórica es el arte de convencer, y en el arte de convencer todo vale. Las estrictas reglas que se aplican a la argumentación científica requieren de unos mecanismos de control extremadamente estrictos para poder mantener su presencia. La superioridad dialéctica en el plano retórico, el reconocimiento por parte del oyente de la superioridad retórica de uno de los participantes de un debate, suele tener un enorme peso a fin de convencer, dado que la experticia informal es una fuente de autoridad (Tyler y Lind 1992). Un orador que basa su estrategia de debate en la superioridad dialéctica es aquel que no busca debatir propiamente con su contrincante, sino aquel que se limita a convencer a la audiencia buscando parecer más inteligente o profundo que su rival. La pseudociencia tiene dos tácticas comunes a fin de desarrollar este tipo de superioridad dialéctica: la logorrea y la descontextualización del lenguaje científico.

La logorrea se podría definir como una tendencia a la verborrea desaforada y sin sentido. Consiste en hablar más, independientemente del sentido o la validez que pueda tener lo dicho. La logorrea es típica de la charlatanería, incluyendo a la pseudociencia (Ladyman 2013), y ha sido durante largo tiempo un gran problema para la filosofía, especialmente dentro de las corrientes posmodernas (Sokal y Bricmont 1999). Un orador logorréico es aquel que, en aras de continuar hablando, apela a falacias de oscuridad constantes (Walton 2002), en un estilo expresivo que supone un torrente de supuesta información semialeatoria que, además de confundir al rival y al oyente, y dadas las condiciones habituales de valoración de la capacidad retórica, hace parecer brillante al hablante cuando su discurso no tiene ni pies ni cabeza. En este sentido, se parece a lo que el término inglés bullshit pretende expresar (Frankfurt 2005): una serie de afirmaciones que, lejos de ser verdaderas o falsas, se desentienden de esa cuestión buscando, simple y llanamente, el ofrecer un mensaje hueco aunque convincente. El hablante medio presenta cierta incapacidad para diferenciar entre las afirmaciones con sentido y el bullshit-pseudoprofundo, una incapacidad que correlaciona positivamente con el pensamiento intuitivo, las creencias paranormales y con el uso de medicina alternativa (Pennycock 2015).

Pero la logorrea pseudocientífica tiene, además, el añadido específico del uso del lenguaje científico de un modo claramente negligente, generando de este modo lo que se suele denominar como «ilusión de profundidad explicativa» (Rozenblit y Keil 2002). Para llevar a cabo esta ilusión entre sus adeptos, la pseudociencia evoluciona en el tiempo buscando adaptarse a las formas lingüísticas que más respeto social posean en un determinado momento. El psicoanálisis hizo lo propio con la termodinámica, durante la New Age fue lo cuántico y lo psico (Rosen 1977), durante cierto tiempo lo bio estuvo de moda, y ahora encontramos una gran cantidad de neuropseudociencia heredada en parte de la New Age (Beyerstein 1990). El caso del lenguaje de la neurociencia es tan potente que incluso podemos ver a otros campos de investigación científica empleando este prefijo pese a que suelen utilizar herramientas propias de la psicología, como el neuromárketing o la neuroeconomía, o pese a llevar a cabo investigaciones propias de la filosofía, como la neuroética. Respecto a la distorsión del lenguaje científico existen casos llamativos por su extremismo, como los enrevesados constructos lingüísticos de la nueva medicina germánica o de la bioneuroemoción, o el de las aparatosas máquinas milagrosas con nombres que explotan el lenguaje de la ciencia de un modo prácticamente caótico, como el «estimulador electro-neural transcutáneo», el «supercargador cerebral» o el «configurador sincro-energizante de ondas ce- 
rebrales» (Tavris 2003). También el uso distorsionado de neuroimágenes o de tablas y fórmulas triviales tiene un gran poder retórico (McCabe y Castel 2008; Tal y Wansink 2016).

\section{FALSO APOyo EXterno, FALSAS CREDENCIALES}

Las personas tienden a mostrar un mayor respeto por las ideas que son apoyadas por una mayoría — un fenómeno habitualmente denominado «efecto bandwagon»-, incluso hasta el nivel de sostener ideas que resultan claramente contraintuitivas o ya refutadas (Bond y Smith 1996). Este tipo de comportamiento responde a las tendencias gregarias prosociales (Baumeister y Leary 1995) y conformistas del ser humano, inhibiendo sus acertadas primeras impresiones, aunque la estrategia adaptativa sea válida en términos de su contexto evolutivo. Pese a que es una actitud que puede arrastrar a la gente hacia cierto conservadurismo doxástico, a priori puede parecer más probable que una idea aceptada por una mayoría resulte más verosímil, dado que, intuitivamente, se tiende a pensar que dicha mayoría habrá hecho una valoración ponderada de la misma. Pero esta idea intuitiva es susceptible de una gran cantidad de matizaciones, dado que los procesos de adopción de creencias no son, ni mucho menos, enteramente racionales.

Un pseudocientífico quiere, además del adoctrinamiento y del refuerzo de grupo, gozar de un apoyo externo que imite de forma adecuada el apoyo externo del que gozan las teorías científicas. Este apoyo externo propio de las teorías científicas se caracteriza por: múltiple evidencia científica, aceptación por parte de la comunidad científica, publicaciones en revistas debidamente indexadas y con altos mecanismos de control, vías específicas de divulgación y presencia en los colegios profesionales y en los contextos sanitarios y educativos estatales - temarios oficiales de institutos y universidades. Para cada uno de estos rasgos encontramos tácticas muy sofisticadas de parasitismo, con situaciones extremas como la venta legal de homeopatía en las farmacias europeas, donde el apoyo externo es casi total en su impostación como ciencia.

Acerca de las publicaciones en revistas exigentes, hay tres estrategias habituales. La primera de ellas consiste en crear revistas carentes de controles rigurosos o directamente carentes de control alguno, con nombres grandilocuentes como el NLP Research Journal, el Interdisciplinary Journal of the International Society of Cryptozoology, o el Journal of Psychiatric Orgone Therapy, entre otros cientos de casos. Una segunda opción consiste en conseguir, por un fallo en los procesos de control de las revistas, publicar un artículo pseudocientífico en una publicación científica. Estos casos son abundantes, con algunos muy sonados como el artículo en The Lancet que relacionaba la vacuna triple vírica con el autismo, y que dio alas al movimiento antivacunas contemporáneo (Wakefield 1998), o las numerosas publicaciones con grandes errores matemáticos que apoyaron el desarrollo de la teoría pseudocientífica Einstein-Cartan-Evans en la prestigiosa revista Foundations of Physics entre 2003 y 2005. ${ }^{1}$

1 Una pseudoteoría formulada por Myron Evans en la cual se anuncia la unificación entre la relatividad general y la mecánica cuántica. Sus ideas han sido duramente criticadas debido a errores matemáticos (Trovon de Carvalho y Rodrigues 2001; Bruhn, 2008; Bruhn, Hehl y Jadczyk, 2008), aunque, en lugar de abandonarlas o reformularlas, Evans continúa publicitándolas, junto a Thomas Bearden, desde el Alpha Institute for Advanced Studies (AIAS), un grupo que, además, afirma desarrollar máquinas de movimiento perpetuo y generadores electromagnéticos sin movimiento. 
A veces es necesario un ojo muy experto y despierto para detectar el fraude, dado que el nivel de sofisticación en los eufemismos puede ser alto. Esos son los casos del clásico fraude publicado en Nature, «Human basophil degranulation triggered by very dilute antiserum against IgE» (Davenas et al. 1988), que consideraba evidencia fraudulenta en favor de la memoria del agua, o «Novel Approach to Activity Evaluation for ReleaseActive Forms of Anti-Interferon-Gamma Antibodies Based on Enzyme-Linked Immunoassay» (Gavrilova et al. 2014), publicado en Plos One, en el cual la homeopatía es denominada «fármacos RA» de manera eufemística. Casos recientes, relacionados también con la homeopatía, consistentes en artículos eufemísticos y engañosos, los hay en revistas como Bulletin of Experimental Biology and Medicine (Emel'yanova 2017) o Neuroscience and Behavioral Physiology (Ganina 2017). Todos estos casos se relacionan estrechamente con el fraude científico, que se calcula afecta al $2 \%$ de las publicaciones en revistas científicas, aunque es muy probable que el número sea aún mayor — cabe mencionar el 33,7\% de malas prácticas en la investigación privada (Fanelli 2009). En estos casos el daño ya está hecho, debido a que, aunque la revista retire dichos artículos, para que ello suceda pueden pasas lustros o incluso décadas - por ejemplo, doce años para retirar el de Wakefield (The Editors Of The Lancet 2010) o seis para los de Evans ('t Hooft 2008) - , y a que, independientemente de que la retirada tenga lugar, la cita se incluirá para siempre en los artículos de las revistas de pseudocientíficos.

Una tercera opción consiste directamente en tener una revista sobre pseudociencia indexada en una base de datos científica de gran prestigio, casos que también son abundantes. Por ejemplo, indexadas en la Web of Science encontramos revistas como International Journal of Psychoanalysis, Homeopathy, Journal of Traditional Chinese Medicine o Journal of Parapsychology.

Muchas veces, sin embargo, basta con mucho menos de lo expuesto hasta ahora. Una bata blanca, parafernalia estereotipada científico-tecnológica o un conjunto de supuestas titulaciones expedidas por centros ajenos a cualquier tipo de oficialidad bastan para convencer a una gran cantidad de personas.

\section{FALSA SUPERIORIDAD MORAL Y EPISTEMOLÓGICA}

Los grupos sociales humanos tienen determinadas dinámicas inherentes, como la jerarquización o el sentimiento de pertenencia. Los pseudocientíficos son un grupo humano que ha ido generando un cierto sentimiento de pertenencia, aunque puedan competir entre ellos. En este sentido, se establecen dos tipos diferenciados de pertenencia. En primer lugar, pertenencia al grupo que defiende una teoría específica, por ejemplo, terapeutas/consumidores de rebirthing, negacionistas de las vacunas o adeptos a la antroposofía. Los defensores de la tierra plana suelen tener serios problemas con los defensores de la tierra hueca, la Gestalt con el psicoanálisis, los homeópatas licenciados en medicina con los que no, etc. Sin embargo, y pese a este clima de ligera competición, las relaciones habituales entre distintas pseudociencias es la mutua colaboración. Muchas de ellas toman prestados conceptos de otras, como la memoria celular - la idea de que todas las células de nuestro cuerpo almacenan memorias, ya sean nuestras o de nuestros antepasados (Smith 1993) - o la represión de memorias - la idea, de origen freudiando (Freud [1896] 1976) y ya refutada (Loftus y Ketcham 1994; McNally 2005), de la represión de recuerdos traumáticos como mecanismo de defensa. Los corpus de ideas pseudocientíficas no suelen tener problemas para tomar 
prestadas ideas de otros corpus diferenciados cuando es necesario, algo que genera un sentimiento de pertenencia colectivo y de mutua colaboración.

Lo que caracteriza el sentimiento de pertenencia a las diversas pseudociencias es, además de un posible negocio y la relación ambivalente con la ciencia, el victimismo y el sentimiento de estar un peldaño por encima de la ciencia «oficial» en un sentido moral y, en ocasiones, epistemológico. Ellos serían la buena ciencia, mientras que sus rivales dentro del sistema serían mala ciencia o, como mucho, ciencia mediocre. Por ello, el problema de la pseudociencia no es propiamente un problema científico, no es una cuestión que se solvente con un debate sobre hechos, dado que las ideas pseudocientíficas, para ser tales, ya han sido refutadas; el problema de la pseudociencia es, en última instancia, un problema de conflicto intergrupal. Es un problema sociológico estrechamente relacionado con la identidad social, en la que un endogrupo choca contra un exogrupo que presenta una identidad diferente. Estas identidades, como es habitual, se han construido con una clara consciencia del «otro», como una comunidad adscrita a una determinada ideología a la que ofrecen su pertenencia intelectual; una constitución ideológico-identitaria que únicamente agrava el problema de la autojustificación ante las refutaciones (Jost, Ledgerwood y Hardin 2008). En el caso de la pseudociencia, el otro, el exogrupo, suelen ser los científicos y no los otros grupos que sostienen creencias sin garantía epistémica - y este es un indicador muy interesante respecto al nivel de autoconciencia de las propios pseudocientíficos respecto al pobre estatus espistemológico de sus ideas.

Por efecto del sesgo endogrupal y del narcisismo colectivo, los grupos tienden a percibirse como moralmente superiores a sus rivales. Este fenómeno se ve especialmente agravado por el estado de disonancia ética de los pseudocientíficos, un estado que los empuja a defender sus acciones éticamente reprobables a la vez que aumentan sus exigencias éticas respecto a las acciones de los demás, declarando sostener un código de comportamiento especialmente exigente (Barkan et al. 2012). De este modo, los pseudocientíficos se caracterizarían por una mayor altura ética que los científicos, incluso en relación a cuestiones ecológicas o políticas. Aunque no se suelen limitar a este mayor nivel ético: muchas veces la pseudociencia se presenta como superior a la propia ciencia que trata de parasitar. Por ejemplo, considerando que su punto de vista es más «holístico», más abarcante, o que ellos comprenden las verdaderas causas de los problemas en lugar de atender a cuestiones superficiales. Existen casos sorprendentes por el nivel de exaltación de la autoconcepción de superioridad respecto a la ciencia, algo especialmente presente cuando se dan arengas nacionalistas dentro de la teoría pseudocientífica, como, por ejemplo, en cierta pseudociencia hinduista (Sokal 2006) que considera que la mecánica cuántica no solo fue ya descrita por los antiguos vedas, sino que fue superada por estos.

La capacidad de seducción de la comunidad de pseudocientíficos, de su identidad social, es aún más sobresaliente si tenemos en cuenta varios factores adicionales. La pseudociencia es una práctica llevada a cabo mayoritariamente por personas con un nivel educativo alto, de estatus socioeconómico medio-alto o alto (MSPSI 2011) y de pertenencia a etnias favorecidas por el sistema social (Chao y Wade 2008) - el mismo sistema social que suele ser ampliamente criticado en el discurso habitual de los pseudocientíficos. Es posible que esto se deba, además de al mayor respeto que una persona con un nivel educativo alto podría presentar hacia la ciencia, al mayor nivel de rebeldía anti-sistema que suelen presentar los grupos sociales que sienten menos dependencia respecto al mismo. En efecto, está ampliamente documentado que los grupos sociales más desfavorecidos son aquellos que justi- 
fican el sistema con mayor vehemencia (Jost et al. 2003; van der Toorn et al. 2015), incluso en situaciones de pobreza extrema (Henry y Saul 2006). Este hecho, sumado a la preferencia social de la que gozan las figuras de autoridad y los grupos sociales pertenecientes a clases altas (Jost, Pelham y Carvallo 2002), genera que la pseudociencia sea aún más atractiva para el grueso de la población.

\section{Conclusiones}

Pese al carácter interdisciplinar del fenómeno de la pseudociencia, la visión psicocognitiva desarrollada en lo anterior se delinea como especialmente relevante a fin de establecer un marco teórico suficientemente potente como para poner en práctica un criterio de demarcación que mejore la calidad de la investigación respecto a la pseudociencia.

Tres serían las conclusiones que se desprenden de lo argumentado:

1. La naturaleza de la pseudociencia consiste en ser un engaño intelectual: en este sentido, puede haber pensamiento paranormal pseudocientífico - parapsicología - o ideas conspirativas que tengas trazas de pseudociencia — como los casos de pseudociencia negacionista. Los mecanismos de impostación como ciencia son la definición misma de la pseudociencia. Para llevar a cabo este proceso, explotan determinadas causas últimas del fenómeno, relacionadas con nuestros sesgos cognitivos y con nuestros mecanismos de vigilancia epistémica, empleando ciertas técnicas que suponen causas próximas del fenómeno - como se ha mencionado: superioridad dialéctica, falso apoyo externo y la falsa superioridad moral y/o epistemológica. Asimismo, el estatus de pseudociencia es variable, matizable y puede ser implícito: el contexto también hace a la pseudociencia. Por ejemplo, una presentación sobre constelaciones familiares llevada a cabo en un centro de crecimiento personal puede presentar unas formas mayoritariamente paranormales, sin embargo, una presentación sobre constelaciones llevada a cabo en una universidad o en un colegio profesional presentará un impacto en los oyentes más relacionado con el que esperaríamos de la pseudociencia. Las prácticas y propuestas teóricas pueden entrar y salir de este estatus.

2. El marco teórico de los estudios sobre la pseudociencia ha de remitirse a estas tácticas de engaño intelectual: que se empleen marcos teóricos que no criban debidamente a la pseudociencia, incluyendo dentro de su clase a otro tipo de creencias carentes de garantía epistémica, supone un grave problema para el avance de nuestro conocimiento respecto a este fenómeno. Las dinámicas psicológicas de la pseudociencia son muy particulares, dado que esta presenta una relación muy compleja y llena de matices respecto al pensamiento analítico y a la ciencia. Todos los estudios llevados a cabo respecto a esta cuestión han de partir de esta premisa teórica, dado que, en caso contrario, el estudio quedaría invalidado al presentar un dominio de estudio demasiado heterogéneo.

3. Los mecanismos de contención de la pseudociencia son complejos y altamente interdisciplinares: la raíz del problema de la pseudociencia se sitúa en una comprensión deficiente respecto a la naturaleza de la autoridad científica, de modo que esta es evaluada apelando a nociones intuitivas o informales de autoridad. Sin embargo, la comprensión de dicha autoridad y su impacto en la presencia de este tipo 
de creencias no ha sido suficientemente estudiada. Además, la creencia en pseudociencia tiene grandes implicaciones sociológicas que se relacionan estrechamente con determinados tipos de pertenencia a grupos sociales, lo cual nos demanda una aproximación de un modo más abarcante que la llevada a cabo hasta ahora.

\section{REFERENCIAS}

't Hooft, Gerardus. 2008. Editorial note. Foundations of Physics 38(1): 1-2.

Alswelmyeen, Monther y Abeer Al olimmat. 2013. The level of understanding of the nature of science for physics teachers and the relationship of that experience with academic qualification. European Scientific Journal 9/5: 15-28.

Anderson, Cameron et al. 2012. A status-enhancement account of overconfidence. Journal of Personality and Social Psychology 103/4: 718-735.

Ariely, Dan, Anat Brach, y Stephan Meier. 2009. Doing Good or Doing Well? Image Motivation and Monetary Incentives in Behaving Prosocially. American Economic Review 99/1: 544-555.

Barkan, Rachel, Shahar Ayal, y Dan Ariely. 2015. Ethical dissonance, justifications, and moral behavior. Current Opinion in Psychology 6: 157-161.

Barkan, Rachel et al. 2012. The pot calling the kettle black: distancing response to ethical dissonance. J Exp Psychol Gen. 141(4): 757-773.

Baumeister, Roy y Mark Leary. 1995. The Need to Belong: Desire for Interpersonal Attachments as a Fundamental Human Motivation. Psychological Bulletin 117/3: 497-529.

Beyerstein, Barry.1990. Brainscams: Neuromythologies of the New Age. International Journal of Mental Health 19/3: 27-36.

Blancke, Stefaan, Maarten Boudry y Massimo Pigliucci. 2016. Why Do Irrational Beliefs Mimic Science? The Cultural Evolution of Pseudoscience. Theoria 83/1: 78-97.

Blancke, Stefaan et al. 2015. Fatal attraction: the intuitive appeal of GMO opposition. Trends in Plant Science 20/7: 414-418.

Bond, Charles y Bella DePaulo. 2006. Accuracy of Deception Judgments. Personality and Social Psychology Review 10/3: 214-234.

Bond, Rod y Peter Smith. 1996. Culture and conformity: A meta-analysis of studies using Asch's (1952b, 1956) line judgment task. Psychological Bulletin 119/1: 111-137.

Boudry, Maarten et al. 2014 What makes weird beliefs thrive? The epidemiology of pseudoscience. Philosophical Psychology 28/8: 1177-1198.

Boudry, Maarten y Johan Braeckman. (2012) How Convenient! The Epistemic Rationale of Self-validating Belief Systems. Philosophical Psychology 25: 341-364.

Braun, Kathryn, Rhiannon Ellis, y Elizabeth Loftus. 2002. Make my memory: how advertising can change our memories of the past. Psychol. Mark. 19: 1-23.

Brotherton, Robert, Chris French y Allan Pickering. 2013. Measuring Belief in Conspiracy Theories: The Generic Conspiracist Beliefs Scale. Frontiers in Psychology 4: 1-15.

Bruhn, Gerhard. 2008. On the Non-Lorentz-Invariance of M.W. Evans' O(3)-Symmetry Law. Foundations of Physics 38/1: 3-6.

Bruhn, Gerhard, Friedrich Hehl, y Arkadiusz Jadczyk. 2008. Comments on «Spin Connection Resonance in Gravitational General Relativity. Acta Phys. Polon. B. 39: 51-58.

Bunge, Mario. 1985. Seudociencia e ideología. Madrid: Alianza.

Chao, MT y CM. Wade. 2008. Socioeconomic Factors and Women's Use of Complementary and Alternative Medicine in Four Racial/Ethnic Groups. Ethn Dis. 18/1: 65-71.

CIS. 2001. Valores y creencias de los jóvenes. http://www.cis.es/cis/export/sites/default/-Archivos/ Marginales/2440_2459/2440/Es2440mar.pdf. Consultado el 16 de abril del 2017. 
Davenas, E et al. 1988. Human basophil degranulation triggered by very dilute antiserum against IgE. Nature 333/6176: 816-8.

Emel'yanova, A et al. 2017 Effects of Release-Active Antibodies to CD4 Receptor on the Level of lck-Kinase in Cultured Mononuclear Cells from Human Peripheral Blood. Bulletin of Experimental Biology and Medicine 162: 323-326.

Epstein, Seymour et al. 1996. Individual differences in intuitive-experiential and analytical-rational thinking styles. Journal of Personality and Social Psychology 71: 390-405.

Fanelli, Daniele. 2009. How Many Scientists Fabricate and Falsify Research? A Systematic Review and MetaAnalysis of Survey Data. PLoS One 4/5: e5738.

Festinger, Leon. 1957 A Theory of Cognitive Dissonance. Stanford, CA: Stanford University Press.

Frankfurt, Harry. 2005. On Bullshit. Princeton: Princeton University Press.

Franz, Timothy y Kris Green. 2013. The impact of an interdisciplinary learning community course on pseudoscientific reasoning in first-year science students. Journal of the Scholarship of Teaching and Learning 13/5: 90-105.

Freud, Sigmund. [1896] 1976. [Zur Ätiologie der Hysterie] La etiología de la histeria. En José Luis Etcheverry ed., Sigmund Freud, obras completas (Volumen 3), 185-218. Buenos Aires: Amorrortu Editores.

Ganina, K. et al. 2017. Release-Active Antibodies to S100 Protein Can Correct the Course of Experimental Allergic Encephalomyelitis. Neuroscience and Behavioral Physiology 47: 163-167.

Gavrilova, Elena et al. 2014. Novel Approach to Activity Evaluation for Release-Active Forms of Anti-Interferon-Gamma Antibodies Based on Enzyme-Linked Immunoassay. PLoS ONE 9/5: e97017.

Gaze, Catherine. 2014. Popular psychological myths: A comparison of students' beliefs across the psychology major. Journal of the Scholarship of Teaching and Learning 14/2: 46-60.

Gervais, Will y Ara Norenzayan. 2012. Analitic Thinking Promotes Religious Disbelief. Science 336/6080: 493-496.

Gino, Francesca y Dan Ariely. 2011. The Dark Side of Creativity: Original Thinkers Can Be More Dishonest. Journal of Personality and Social Psychology.

Gino, Francesca, Shahar Ayal, y Dan Ariely. 2009. Contagion and differentiation in unethical behavior: the effect of one bad apple on the barrel. Psychol Sci. 20/3: 393-398.

-. 2013. Self-Serving Altruism? The Lure of Unethical Actions that Benefit Others. J Econ Behav Organ. 93: 285-292.

Goldman, Alvin. 2001. Experts: Which Ones Should You Trust?. Philosophy and Phenomenological Research 63/1: 85-110.

Hansson, Sven Ove. 2009. Cutting the Gordian Knot of Demarcation. International Studies in the Philosophy of Science 23/3: 237-243.

-. 2013. Defining pseudoscience and science. In Massimo Pigliucci and Maarten Boudry eds., Philosophy of Pseudoscience: Reconsidering the Demarcation Problem, 61-77. Chicago: University of Chicago Press.

-. 2017. Science denial as a form of pseudoscience. Submitted manuscript.

Henry, P. y A. Saul. 2006. The Development of System Justification in the Developing World. Social Justice Research 19/3: 365-37.

Hughes, Sean, Fiona Lyddy y Sinead Lambe. 2013. Misconceptions about Psychological Science: a review. Psychology Learning and Teaching 12/1: 20-31.

Johnson, Matthew y Massimo Pigliucci. 2004. Is Knowledge of Science Associated with Higher Skepticism of Pseudoscientific Claims?. The American Biology Teacher 66/8: 536-548.

Jost, John et al. 2003. Social inequality and the reduction of ideological dissonance on behalf of the system: evidence of enhanced system justification among the disadvantaged. Eur. J. Soc. Psychol. 33: 13-36.

Jost, John, Allison Ledgerwood, y Curtis Hardin. 2008. Shared reality, system justification, and the relational basis of ideological beliefs. Social and Personality Psychology Compass 2: 171-186.

Jost, John, Brett Pelham y Mauricio Carvallo. 2002. Non-conscious forms of system justification: Cognitive, affective, and behavioral preferences for higher status groups. Journal of Experimental Social Psychology 38: 586-602. 
Kaikati, J. 1987. Celebrity Advertising. A Review and Synthesis. International Journal of Advertising 6/2: 93-105.

Ladyman, James. 2013. Toward a Demarcation of Science from Pseudoscience. In Massimo Pigliucci and Maarten Boudry eds., Philosophy of Pseudoscience: Reconsidering the Demarcation Problem, 45-59. Chicago: University of Chicago Press.

Lamba, Shakti y Vivek Nityananda. 2014. Self-Deceived Individuals Are Better at Deceiving Others. PLoS One 9/8: e104562.

Levine, Timothy. 2010. A Few Transparent Liars: Explaining 54\% Accuracy in Deception Detection Experiments. Annals of the International Communication Association 34/1: 41-61.

- 2011. Sender Demeanor: Individual Differences in Sender Believability Have a Powerful Impact on Deception Detection Judgments. Human Communication Research 37/3: 377-403.

-. 2014. Truth-Default Theory (TDT): A Theory of Human Deception and Deception Detection. Journal of Language and Social Psychology 33/4: 378-392.

Levine, Timothy, Rachel Kim, y Lauren Hamel. 2010. People Lie for a Reason: Three Experiments Documenting the Principle of Veracity. Communication Research Reports 27/4: 271-285.

Lewandowsky, Stephan, Gilles Gignac, y Klaus Oberauer. 2013. The role of conspiracist ideation and worldviews in predicting rejection of science. PLoS One 8/10: e75637.

Lewandowsky, Stephan, Klaus Oberauer, y Gilles Gignac. 2013. NASA Faked the Moon Landing-Therefore, (Climate) Science Is a hoax. An Anatomy of the Motivated Rejection of Science. Psychological Science 24/5: 622-633.

Lilienfeld, Scott, Jeffrey Lohr, y Dean Morier. 2004. The Teaching of Courses in the Science and Pseudoscience of Psychology: Useful Resources. Teaching of Psychology 28/3: 182-191.

Lindeman, Maarjana y Kia Aarnio. 2006. Paranormal beliefs: Their dimensionality and correlates. European Journal of Personality 20: 585-602.

Lobato, Emilio et al. (2014) Examining the Relationship Between Conspiracy Theories, Paranormal Beliefs, and Pseudoscience Acceptance Among a University Population. Applied Cognitive Psychology 28: 617-625.

Loftus, Elizabeth y Katherine Ketcham. 1994. The Myth of Repressed Memory. NY: St. Martin's Press.

Lord, Charles, Lee Ross y Marc Lepper. 1979. Biased assimilation and attitude polarization: The effects of prior theories on subsequently considered evidence. Journal of Personality and Social Psychology 37/11: 2098-2109.

Losh, Susan y Brandon Nzekwe. 2011. Creatures in the classroom: Preservice teacher beliefs about fantastic beasts, magic, extraterrestrials, evolution, and creation. Science \& Education 20/5: 473-489.

Lundstrôm, Mats y Anders Jakobsson. 2009. Students' Ideas Regarding Science and Pseudo-science in Relation to the Human Body and Health. Nordina 5/1:3-17.

Majima, Yoshimasa. 2015. Belief in Pseudoscience, Cognitive Style and Science Literacy. Applied Cognitive Psychology 29: 552-559.

Mann, Heather et al. 2016. Cut from the Same Cloth. Similarly Dishonest Individuals Across Countries. Journal of Cross-Cultural Psychology 47/6: 858-874.

Mascaro, Olivier y Dan Sperber. 2009. The Moral, Epistemic, and Mindreading Components of Children's Vigilance towards Deception. Cognition 112/3: 367-380.

Mazar, Nina, On Amir, y Dan Ariely. 2008. The Dishonesty of Honest People: A Theory of Self-Concept Maintenance. Journal of Marketing Research 45/6: 633-644.

McCabe, David y Allan Castel. 2008. Seeing is believing: The effect of brain images on judgments of scientific reasoning. Cognition 107/1:343-352.

McNally, Richard. 2005. Debunking Myths about Trauma and Memory. The Canadian Journal of Psychiatry 50/13: 817-822.

Metzger, Miriam y Andrew Flanagin. 2013. Credibility and trust of information in online environments: The use of cognitive heuristics. Journal of Pragmatics 59: 210-220.

Michener, Andrew y Martha Burt, 1975. Components of «authority» as determinants of compliance.J Pers Soc Psychol. 31/4:606-614. 
Milgram, Stanley. 1963. Behavioral study of obedience. J Abnorm Soc Psychol 67: 371-378.

MSPSI. 2011. Análisis de situación de las terapias naturales. http://www.mspsi.gob.es/novedades/docs/analisisSituacionTNatu.pdf. Consultado el 18/04/2017.

Murphy, Sean et al. 2015. The Role of Overconfidence in Romantic Desirability and Competition. Pers Soc Psychol Bull. 41/8: 1036-1052.

Nyhan, Brendan y Jason Reifler. 2015. Does correcting myths about the flu vaccine work? An experimental evaluation of the effects of corrective information. Vaccine 3/3: 459-464.

Park, Hee Sun et al. 2010. How people really detect lies. Communication Monographs 69/2: 144-157.

Pennycock, Gordon et al. 2015. On the reception and detection of pseudo-profound bullshit. Judgment and Decision Making 10/6: 549-563.

Pigliucci, Massimo. 2007. The evolution-creation wars: why teaching more science just is not enough. McGill Journal of Education 42/2: 285-306.

-. 2012. The Demarcation Problem. A (Belated) Response to Laudan. In Massimo Pigliucci and Maarten Boudry eds., Philosophy of Pseudoscience: Reconsidering the Demarcation Problem, 9-28. Chicago: University of Chicago Press.

Praxmarer, Sandra. 2011. How a presenter perceived attractiveness affects persuasion for attractiveness-unrelated products. International Journal of Advertising 30/5: 839-865.

Quandt, Sara et al. 2009. Development of an International Questionnaire to Measure Use of Complementary and Alternative Medicine (I-CAM-Q). The Journal of Alternative and Complementary Medicine 15/4: 331-339.

Roediger, Henry y Kathleen McDermott. 1995. Creating False Memories: Remembering Words Not Presented in Lists. Journal of Experimental Psychology: Learning, Memory, and Cognition 4/21: 803-814.

Ronay, Richard et al. 2016. Embodied power, testosterone, and overconfidence as a causal pathway to risktaking. Comprehensive Results in Social Psychology 1-16.

Rosen, Richard. 1977. Psychobabble: Fast Talk and Quick Cure in the Era of Feeling. New York: Atheneum.

Rozenblit, Leonid y Frank Keil. 2002. The misunderstood limits of folk science: an illusion of explanatory depth. Cognitive Science 26/5: 521-562.

Schmidt K., R. Levenstein y Z. Ambadar. 2012. Intensity of smiling and attractiveness as facial signals of trustworthiness in women. Percept Mot Skills. 114/3: 964-78.

Serota, Kim y Timothy Levine. 2014. A Few Prolific Liars. Variations in the Prevalence of Lying. Journal of Language and Social Psychology 34/2: 138-157.

Serota, Kim, Timothy Levine, y Franklin Boster. 2010. The Prevalence of Lying in America: Three Studies of Self-Reported Lies. Human Communication Research 36/1: 2-25.

Smith, Susan. 1993. Body Memories: And Other Pseudo-Scientific Notions of «Survivor Psychology». Issues in Child Abuse Accusations 5/4: 220-234.

Smith, Megan, Robert Trivers, y William von Hippel. 2017. Self-deception facilitates interpersonal persuasion. Journal of Economic Psychology (In press).

Sokal, Alan. 2006. Pseudoscience and Postmodernism: Antoginists or fellow-travellers? In Garrett Fagan ed., Archaeological Fantasies: How Pseudoarchaeology Misrepresents the Past and Misleads the Public, 286-361. New York: Routledge.

Sokal, Alan y Jean Bricmont. 1999. Imposturas Intelectuales. Barcelona: Paidós.

Sperber Dan et al. 2010. Epistemic Vigilance. Mind \& Language 25/4: 359-393.

Sumranwanich, Wimol y Chokchai Yuenyong. 2014. Graduate Students' Concepts of Nature of Science (NOS) and Attitudes toward Teaching NOS. Procedia - Social and Behavioral Sciences 116: 2443-2452.

Steele, Claude. 1988. The psychology of self-affirmation: Sustaining the integrity of the self. Advances in experimental social psychology 21:261-302.

Schwartz, Janet, Mary Luce, y Dan Ariely. 2011. Are Consumers Too Trusting? The Effects of Relationships with Expert Advisers. Journal of Marketing Research 48: 163-174.

Tal, Aner y Brian Wansink. 2016. Blinded with science: Trivial graphs and formulas increase ad persuasiveness and belief in product efficacy. Public Understanding of Science 25/1: 117-125. 
Tavris, Carol. 2003. Foreword. In Scott Lilienfeld, Steven Lynn and Jeffrey Lohr eds., Science and pseudoscience in clinical psychology, IX-XVIII. New York: The Guilford Press.

The Editors Of The Lancet. 2010. Retraction - Ileal-lymphoid-nodular hyperplasia, non-specific colitis, and pervasive developmental disorder in children. The Lancet 375/9713: 445.

The Global Deception Research Team. 2006. A World of Lies. Journal of Cross-Cultural Psychology 37/1: 60-74.

Tobacyk, Jerome. 2004. A Revised Paranormal Belief Scale. International Journal of Transpersonal Studies 23/1: 94-98.

Trovon de Carvalho, Alexandre Luis y Waldyr Rodrigues. (2001). The Non Sequitur Mathematics and Physics of the «New Electrodynamics» Proposed by the AIAS Group. Random Operators and Stochastic Equations 9/2: 161-206.

Tsang, Jo-Ann. 2002. Moral rationalization and the integration of situational factors and psychological processes in immoral behavior. Review of General Psychology 6/1:25-50.

Tseng, Yuan-Chueh et al. 2014 The Relationship Between Exposure to Pseudoscientific Television Programmes and Pseudoscientific Beliefs among Taiwanese University Students. International Journal of Science Education B/4: 107-122.

Tsujii, Takeo y Shigeru Watanabe. 2009. Neural correlates of dual-task effect on belief-bias syllogistic reasoning: a near-infrared spectroscopy study. Brain Research 1287: 118-125.

Tyler, Tom. 2006. Psychological Perspectives on Legitimacy and Legitimation. Annu. Rev. Psychol. 57: 375400.

Tyler, Tom y Allan Lind. 1992. A Relational Model of Authority in Groups. Advances in Experimental Social Psychology 25: 115-191.

van der Toorn, Jojanneke et al. 2015. A Sense of Powerlessness Fosters System Justification: Implications for the Legitimation of Authority, Hierarchy, and Government. Political Psychology 36/1: 93-110.

van der Toorn, Jojanneke, Tom Tyler, y John Jost. 2011. More than fair: Outcome dependence, system justification, and the perceived legitimacy of authority figures. Journal of Experimental Social Psychology 47/1: 127-138.

von Hippel, William y Robert Trivers. 2011. The evolution and psychology of self-deception. Behavioral and Brain Sciences 34: 1-56.

Wakefield, Andrew et al. 1998. Ileal-lymphoid-nodular hyperplasia, non-specific colitis, and pervasive developmental disorder in children. The Lancet 351/9103: 637-41.

Walker, Richard et al. 2002. Science Education Is No Guarantee of Skepticism. Skeptic 9/3: 24-27.

Walton, Douglas. 2002. Fallacies Arising from Ambiguity. Dordrecht: Kluwer Academic Publishers.

Angelo Fasce es doctorando en Lógica y Filosofía de la Ciencia en la Universitat de València. Su campo de investigación es el criterio de demarcación y la pseudociencia como fenómeno psicológico. Es, además, un activo divulgador de la ciencia y de su filosofía.

Dirección: Universidad de Valencia (Departamento de Filosofía) Avd. Blasco Ibañez, 30. 46010 ValenciaEspaña. Email: angelofasce@hotmail.com 